\title{
Correspondence
}

\section{Ideology over evidence?}

In her narrative, Dr Moncrieff makes assertions about depressive illness, antidepressants, and psychotropic medications. ${ }^{1}$ Her main points are that these medications are not clinically effective when using rating scales, and that the models proposed for antidepressant action are erroneous. We would suggest that the narrative reflects ideology, as opposed to evidence, and should be interpreted accordingly.

First, a 1969 narrative supplementary review is given as an example of the lack of efficacy of tricyclic and older antidepressants. A more recent (and comprehensive) review found significant benefits for monoamine oxidase inhibitors over placebo, which were surpassed by tricyclics. ${ }^{2}$ The argument is then made that changes on the Hamilton Rating Scale for Depression (HRSD) are minimal, in comparison with placebo, and that differences are clinically insignificant when the Clinical Global Impression (CGI) scale is used, citing among other reviews the Kirsch meta-analysis (where the effect size was $0.31^{3}$ ). A similar effect size was seen in a recent analysis of over 500 studies, which reported odds ratios of between 1.37 and 2.13 for response compared with placebo. ${ }^{4}$

In focusing the argument on change in total HRSD score, Dr Moncrieff appears unaware that the scale was never intended to measure change. A more robust way of analysing it was recently demonstrated, using the rating of subjective mood (item 1 on the HRSD), which would be akin to the CGI. This avoided the influence of antidepressant side-effects on the scale, and found clear benefits for paroxetine and citalopram over placebo. ${ }^{5}$

A study cited to indicate severity of depression did not predict outcome, evaluated the short-term efficacy of antidepressants and was not intended to test the hypothesis of severity, with the authors reporting significant benefits of fluoxetine over placebo in adults (improvement of approximately 35\%). ${ }^{6}$ The 1964 Medical Research Council trial (which showed the efficacy of electroconvulsive therapy) is given as evidence of lack of effect of severity on response; however, the statement that antidepressants did not outperform placebo is not surprising, given that the dose of imipramine was $50 \mathrm{mg}$ and that of phenelzine $15 \mathrm{mg}$. A more recent and influential publicly funded study (cited over 3000 times in Google Scholar) showed the effectiveness of imipramine (at a therapeutic dose of around $185 \mathrm{mg}$ ) in people with severe depression, in comparison with psychological therapies (cognitive-behavioural therapy and interpersonal therapy). These therapies showed little benefit over placebo in this group. ${ }^{7}$

The rest of the narrative dwells on 'disease-centred' models of psychiatric illness, as an alternative to the current 'targeting a brain abnormality' approach. We are unaware of modern psychiatry relying on the neurotransmitter models she discusses; the field has moved on significantly, and most neuroscientists would point to more nuanced models involving effects on neural networks and plasticity. ${ }^{8}$ The predominant references cited here are Dr Moncrieff's own hypotheses.

In summary, we would suggest that Dr Moncrieff's narrative is selective at best, and on cursory examination there is little effort to appraise the literature in a scientifically objective manner. One cannot help but assume that this opinion piece represents ideology over evidence, and therefore any interpretation should be cautious.

\section{Declaration of Interests}

Professor Young has the following disclosures: Employed by King's College London; Honorary Consultant SLaM (NHS UK); paid lectures and advisory boards for the following companies with drugs used in affective and related disorders: Astrazenaca, Eli Lilly, Lundbeck, Sunovion, Servier, Livanova, Janssen; No share holdings in pharmaceutical companies; lead Investigator for Embolden Study (AZ), BCI Neuroplasticity study and Aripiprazole Mania Study; Investigator initiated studies from AZ, Eli Lilly, Lundbeck, Wyeth, Janssen. Grant funding (past and present): NIMH (USA); CIHR (Canada); NARSAD (USA); Stanley Medical Research Institute (USA); MRC (UK); Wellcome Trust (UK); Royal College of Physicians (Edin); BMA (UK); UBC-VGH Foundation (Canada); WEDC (Canada); CCS Depression Research Fund (Canada); MSFHR (Canada); NIHR (UK). Janssen (UK)

Sameer Jauhar, Senior Research Fellow, and Allan H. Young, Director, Centre for Affective Disorders, Institute of Psychiatry, Psychology and Neuroscience, King's College, London; Research Fellow, Centre for Affective Disorders, Institute of Psychiatry, Psychology and Neuroscience, King's College; email: sameer.jauhar@kcl.ac.uk

1 Moncrieff J. Antidepressants are not antidepressants - an alternative approach to drug action and implications for the use of antidepressants. BJPsych Bull 2018; 42(1): 42-4.

2 Thase ME, Trivedi MH, Rush AJ. MAOls in the contemporary treatment of depression. Neuropsychopharmacology 1995; 12(3): 185-219.

3 Kirsch I, Deacon BJ, Huedo-Medina TB, Scoboria A, Moore TJ, Johnson BT. Initial severity and antidepressant benefits: a meta-analysis of data submitted to the Food and Drug Administration. PLoS Med 2008; 5(2): e45.

4 Cipriani A, Furukawa TA, Salanti G, Chaimani A, Atkinson LZ, Ogawa Y, et al. Comparative efficacy and acceptability of 21 antidepressant drugs for the acute treatment of adults with major depressive disorder: a systematic review and network meta-analysis. Lancet 2018; 391(10128): 1357-66.

5 Hieronymus F, Lisinski A, Nilsson S, Eriksson E. Efficacy of selective serotonin reuptake inhibitors in the absence of side effects: a mega-analysis of citalopram and paroxetine in adult depression. Mol Psychiatry 2017; doi: 10.1038/mp.2017.147.

6 Gibbons RD, Hur K, Brown CH, Davis JM, Mann JJ. Benefits from antidepressants: synthesis of 6-week patient-level outcomes from doubleblind placebo-controlled randomized trials of fluoxetine and venlafaxine. Arch Gen Psychiatry 2012; 69(6): 572-9.

7 Elkin I, Shea MT, Watkins JT, Imber SD, Sotsky SM, Collins JF, et al. National Institute of Mental Health Treatment of Depression 
Collaborative Research Program: general effectiveness of treatments. Arch Gen Psychiatry 1989; 46(11): 971-82.

8 Morrison PD, Murray RM. The antipsychotic landscape: dopamine and beyond. Ther Adv Psychopharmacol 2018; 8(4): 127-35.

doi:10.1192/bjb.2018.32

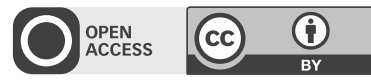

(c) The Authors 2018. This is an Open Access article, distributed under the terms of the Creative Commons Attribution licence (http://creativecommons.org/ licenses/by/4.0/), which permits unrestricted re-use, distribution, and reproduction in any medium, provided the original work is properly cited.

\section{Rational antidepressant use}

In her contribution to the 'Against the Stream' series,

Dr Moncrieff ${ }^{1}$ articulates the case for the drug-centred model of antidepressant action. She notes that antidepressants do not typically outperform placebo in well-designed studies (particularly in rare instances where an active placebo is used as a control $^{2}$ ), have little clinical effect and can cause serious adverse effects. Having made the case that antidepressants are not 'specific' antidepressant agents, she makes some comments about their use in clinical practice. I would like to offer a few remarks about these issues, including some musings about what 'rational antidepressant use' might look like.

Modern psychiatric practice has seen the rise and fall of several promising antidepressant agents (the monoamine oxidase inhibitors, the tricyclic antidepressants and selective serotonin reuptake inhibitors (SSRIs)). Recent efforts include testing the possible antidepressant properties of ketamine. But are these efforts futile? Perhaps yes, perhaps no. A truly specific antidepressant drug (if one is ontologically possible) appears to be a pipedream, given current diagnostic limitations. Our categorisation of major depressive disorder is highly heterogeneous, ${ }^{3}$ creating a disjunctive category of cognitive, behavioural and biological symptoms that do not reliably cluster together. Even if any of our current drugs had specificity for 'depression', this would be extremely difficult to uncover in clinical practice or research settings. As a result, drug development will be prone to ideological, as opposed to scientific, revolutions. ${ }^{4}$

Should we therefore abandon antidepressants as a treatment modality? As long as we are honest with our patients about our current state of knowledge, I think not. Drug use has always been an integral part of human life, ${ }^{5}$ helping to alleviate life's various physical, emotional and existential pains. Antidepressants are no different in this respect. While researchers continue the search for a discrete condition called 'depression', drugs such as the SSRIs can be exploited for particular patient complaints. Antidepressants can cause emotional blunting, sedation, activation and decreased libido, among other things. Some have a proclivity towards one effect more than others. These effects can be exploited to relieve particular problems (e.g. sedation to alleviate insomnia, or emotional numbing to transcend an episode of intense anxiety or distress), without pretence towards a yet-to-be discovered condition. A rational provider would match a drug's effects to the patient's complaints, irrespective of diagnosis (or drug class); and would remain vigilant to the development of any adverse effects or deterioration of condition, start at the lowest recommended dose, and withdraw the patient from the drug as soon as possible. Psychosocial interventions can remain an important part of treatment, in many cases being the first treatment of choice. Antidepressants, like all drugs, are neither angels nor demons. They should be used selectively and thoughtfully, when used at all.

Daniel Dunleavy, MSW, Doctoral Candidate, Florida State University College of Social Work; email: djd09e@fsu.edu

1. Moncrieff J. Antidepressants are not antidepressants - an alternative approach to drug action and implications for the use of antidepressants. BJPsych Bull 2018; 42(1): 42-4.

2. Jensen JS, Bielefeldt AS, Hróbjartsson A. Active placebo control groups of pharmacological intervention were rarely used but merited serious consideration: a methodological overview. J Clin Epidemiol 2017; 87: 35-46.

3. Paris J. The mistreatment of major depression. Can J Psychiatry 2014; 59 (3): 148-51.

4. Healy DT. The structure of psychopharmacological revolutions. Psychiatr Dev 1987; 4: 349-76.

5. Chast F. A history of drug discovery. In The Practice of Medicinal Chemistry (ed CG Wermuth), 3rd edn. Academic Press, 2008.

doi:10.1192/bjb.2018.33

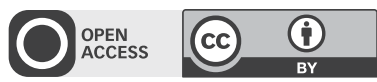

(c) The Author 2018. This is an Open Access article, distributed under the terms of the Creative Commons Attribution licence (http://creativecommons.org/ licenses/by/4.0/), which permits unrestricted re-use, distribution, and reproduction in any medium, provided the original work is properly cited.

Author's reply: In response to Dr Jauhar and Professor Young, I am used to being accused of using ideology, of being selective, of not being balanced or of being polemical. I take no personal offence, but it is important to point out that this is a useful tactic if you want to shut down debate. It harnesses the authority of science to present one view as neutral, objective and credible, and the other as self-interested and unreliable. In truth, we all bring assumptions and biases to our work. I am obviously unable to describe every study ever done on antidepressants in a short article, but I have written books and papers that address all the evidence I could find that supports the disease-centred model of drug action in relation to antidepressants and other psychiatric drugs. ${ }^{1}$

Indeed, one of the most important points I am making in relation to drug action is that existing psychopharmacological research is based on unexamined assumptions about how drugs work. These consist of the idea that drugs target the neurological mechanisms underlying symptoms, whether the latest theory about mechanisms concerns abnormalities of neurotransmitters, neural networks or neuro-plasticity. This idea has allowed psychopharmacology research to ignore the alterations to normal functioning that psychiatric drugs produce, and that will affect mental states including mental disorders, regardless of the underlying mechanisms.

Jauhar and Young point out that the latest meta-analysis of antidepressant trials finds impressive odds ratios for effects of antidepressants, but it analyses categorical outcomes derived 